\title{
Comments on "An update of the HLS estimate of the muon g-2" by M. Benayoun et al., EPJC (2013) 73:2453
}

\author{
M. Davier ${ }^{1, \mathrm{a}}$, B. Malaescu ${ }^{2, \mathrm{~b}}$ \\ ${ }^{1}$ Laboratoire de l'Accélérateur Linéaire, IN2P3-CNRS et Université de Paris-Sud, 91898 Orsay, France \\ ${ }^{2}$ Laboratoire de Physique Nucléaire et des Hautes Energies, IN2P3-CNRS et Universités Pierre-et-Marie-Curie et Denis-Diderot, 75252 Paris \\ Cedex 05, France
}

Received: 28 June 2013 / Revised: 13 August 2013 / Published online: 13 November 2013

(C) The Author(s) 2013. This article is published with open access at Springerlink.com

\begin{abstract}
In a recent paper, Benayoun et al. (Eur. J. Phys. C $73: 2453,2013)$ use a specific model to compare results on the existing data for the cross section of the process $e^{+} e^{-} \rightarrow \pi^{+} \pi^{-}$and state conclusions about the inconsistency of the BABAR results with those from the other experiments. We show that a direct model-independent comparison of the data at hand contradicts this claim. Clear discrepancies with the results of Benayoun et al. (Eur. J. Phys. C 73:2453, 2013) are pointed out. As a consequence we do not believe that the lower value and the smaller uncertainty obtained for the prediction of the muon magnetic anomaly are reliable results.
\end{abstract}

\section{Introduction}

The authors of Ref. [1] use a specific model of the cross section for the process $e^{+} e^{-} \rightarrow \pi^{+} \pi^{-}$in order to compute the corresponding contribution to the muon magnetic anomaly. Specifically they fix parameters of a theoretical model based on the concept of Hidden Local Symmetry (references to be found in Ref. [1]) using the spectral function from $\tau$ decays obtained by the Belle collaboration [2] and other less precise inputs from additional processes assuming vector dominance.

In these comments we consider four points relevant to the analysis in Ref. [1] where the model is used to discriminate between the different sets of recent $e^{+} e^{-}$data, allowing the authors to make statements about their consistency.

First, their model for the $e^{+} e^{-}$cross section relies on the $\tau$ spectral function from Belle within the framework of the HLS approach. Such a procedure relies on assumptions regarding the isospin-breaking corrections needed in order to

\footnotetext{
a e-mail: davier@lal.in2p3.fr

be-mail: malaescu@in2p3.fr
}

link the $\tau$ and $e^{+} e^{-}$spectral functions. We provide some comments on this point in Sect. 2.

Second, the model is used by the authors to compare the results of the model with existing measurements of the $\pi^{+} \pi^{-}$annihilation process. In the peak $\rho$ region they claim good agreement between the data from KLOE [3, 4], CMD2 [5, 6], and SND [7], while pointing out a discrepancy with BABAR [8, 9]. As we show in Sect. 3 this conclusion is not consistent with the direct comparison of these data.

Third, still using the model, a specific analysis of the $\rho-\omega$ interference pattern in the above experimental data is performed, again showing an even bigger discrepancy between BABAR and the other experiments. However the $\rho-\omega$ interference has been already extracted by the experiments (but KLOE) and the published results clearly do not support the claim made by the authors, as demonstrated in Sect. 4.

Fourth, we comment on the values obtained for the hadronic contribution to the muon $g-2$ and on the stated increased significance of the discrepancy with the direct measurement [10] which we believe is not warranted.

In the following we address these four issues. For the second and third points, we show that in a model-independent way the direct comparison of the measured cross sections yields results which are at variance with those of Ref. [1].

\section{Isospin breaking corrections}

In addition to the standard isospin-breaking (IB) corrections [11-18], M. Benayoun et al. consider the effect of $\gamma-\rho$ mixing, already proposed previously [19]. The effect of mixing is not shown quantitatively in Ref. [1], but from the results in Ref. [19] one can observe that a major consequence in the $\rho$ region is a mass shift between neutral and charged $\rho$ resonances, although some additional effects 
are seen in the tails. A mass shift was already considered in the analysis of Ref. [18], together with electromagnetic contributions in the $\rho$ decays, with the conclusion that the isospin-corrected $\tau$ spectral function agreed well with the BABAR data and rather well with the Novosibirsk results, while disagreeing with KLOE.

The authors of Ref. [1] reach the opposite conclusion, i.e. good agreement with KLOE, fair agreement with CMD-2 and SND, and discrepancy with BABAR. We point out that even if the corrections used follow the same rules as in Ref. [19], their final results differ significantly. Therefore it seems that the IB corrections as used by the authors are still subject to some debate, especially since they do not provide specific studies of their systematic uncertainties. In these conditions it is probably not safe to use the IB-corrected $\tau$ spectral function as an exact replica of the $e^{+} e^{-}$cross section when comparing to the $e^{+} e^{-}$data.

\section{Consistency of different data in the peak $\rho$ region}

The direct comparison of the cross sections for BABAR and the other experiments has been shown in detail in Ref. [9]. There is indeed a discrepancy with KLOE, but agreement with CMD-2 and SND within the quoted respective systematic uncertainties. One can quantify the observations by considering the ratio of the cross sections measured by each experiment to the BABAR result. Since the energy corresponding to the experimental data points do not match the BABAR bins, we perform the quantitative comparison to the form factor fit of the BABAR data, described in Ref. [9] which nicely interpolates the data.

From inspection of their Fig. 5 the authors of Ref. [1] point out major differences in the $\rho$ peak region 0.70 $0.85 \mathrm{GeV}$. Thus we restrict the ratio fits to this mass range. We exclude the $\rho-\omega$ region $0.776-0.788 \mathrm{GeV}$, discussed in the next section, as the ratios are very sensitive to the relative mass calibration of the experiments (this effect is not taken into account in Ref. [1]). The ratios to BABAR as a function of $\pi \pi$ mass are displayed in Fig. 1. The fit to a constant is a good description of the respective data, except for KLOE where deviations are seen at lower masses, however not affecting significantly the fit over most of the mass range.

The fitted values for the ratios are given in Table 1 . Of course the BABAR value in the first row is very close to one by construction, but we show it to demonstrate that the form factor fit, performed in the full mass range from threshold to $3 \mathrm{GeV}$, does represent well the BABAR data between 0.7 and $0.85 \mathrm{GeV}$. Only statistical uncertainties are used in the ratio fits, while systematic uncertainties are quoted separately. All the values are displayed in Fig. 2.

From Table 1 and Fig. 2 we see that, unlike the qualitative claim in Ref. [1], the BABAR data in the $\rho$ peak region is fully compatible with CMD-2 and SND, and disagrees with KLOE. The CMD-2 data also disagrees with KLOE, while SND with a larger uncertainty is consistent with both BABAR and KLOE. Since in Ref. [1] CMD-2 and SND are treated together, it is interesting to quote the ratio for the combined Novosibirsk data (taking into account correlated uncertainties). Its value of $0.999 \pm 0.007$ shows the same disagreement with KLOE as BABAR. Therefore we conclude that the claim by Benayoun et al. is incorrect. There is no justified argument for discarding the BABAR data in the evaluation of the $g-2$ dispersion integral.

\section{Analysis of $\rho-\omega$ interference}

Except for KLOE, all the experiments have provided fits of the pion form factor with $\rho$ and $\omega$ amplitudes, even including isovector resonances at higher mass. In these analyses the parameters describing the $\rho$ resonance and the interference amplitude are fitted, so that the product of the branching ratios $B_{\omega \rightarrow e e} \cdot B_{\omega \rightarrow \pi \pi}$ can be directly obtained. Using the fit results published by the experiments, the values deduced for the product are given in Table 2 . The values presented in Ref. [1] are listed for comparison. The agreement is reasonable for CMD-2 and SND, however there is a large
Table 1 Ratios of the measured $e^{+} e^{-} \rightarrow \pi^{+} \pi^{-}$cross sections from the quoted experiments relative to the BABAR form factor fit in the mass range $0.7-0.85 \mathrm{GeV}$, excluding the $\rho-\omega$ interference region.
The quoted uncertainties are statistical ('stat'), systematic ('syst'), and total ('tot'). The $\chi^{2}$ per degree of freedom $(D F)$ of the ratio fits to a constant are given

\begin{tabular}{|c|c|c|c|c|c|}
\hline Experiment/BABAR-fit & ratio & 'stat' & $\chi^{2} / D F$ & 'syst' & 'tot' \\
\hline BABAR & 0.999 & 0.001 & $52.5 / 60$ & 0.005 & 0.005 \\
\hline CMD-2 2003 & 1.002 & 0.007 & $18.6 / 17$ & 0.006 & 0.009 \\
\hline CMD-2 2006 & 1.001 & 0.004 & $6.1 / 11$ & 0.008 & 0.009 \\
\hline SND & 0.991 & 0.002 & $9.4 / 11$ & 0.013 & 0.013 \\
\hline KLOE 2008 & 0.976 & 0.001 & $66.8 / 20$ & 0.009 & 0.009 \\
\hline KLOE 2010 & 0.974 & 0.002 & $39.8 / 20$ & 0.008 & 0.008 \\
\hline
\end{tabular}



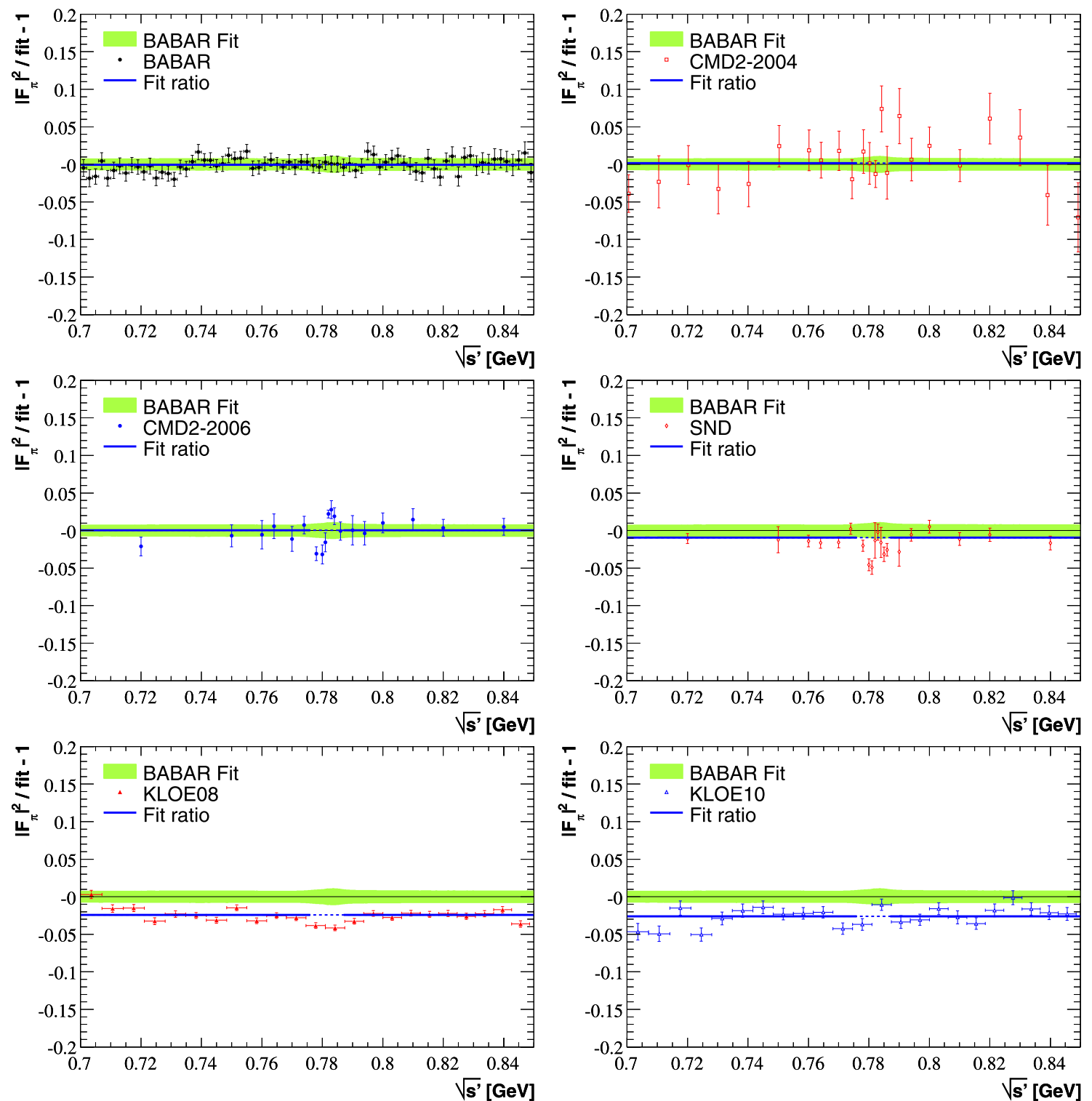

Fig. 1 Fits to a constant of the ratios (minus 1) of the measured $e^{+} e^{-} \rightarrow \pi^{+} \pi^{-}$cross sections from the quoted experiments relative to the BABAR cross section fit in the mass range $0.7-0.85 \mathrm{GeV}$, excluding the $\rho-\omega$ interference region. Only statistical uncertainties are used

in the fits and plotted. The result of the fits is shown with a blue line, while the green band around 0 gives the region allowed by BABAR statistical and systematic uncertainties (Color figure online)

Table 2 The results for the product $B_{\omega \rightarrow e e} \cdot B_{\omega \rightarrow \pi \pi}$ obtained from pion form factor fits published by the experiments are compared to the results presented in Ref. [1]. The conclusions regarding the BABAR value and its uncertainty are radically different in the two cases

\begin{tabular}{lll}
\hline Experiment & $B_{e e}^{\omega} \cdot B_{\pi \pi}^{\omega}\left(10^{-6}\right)[\exp ]$ & $B_{e e}^{\omega} \cdot B_{\pi \pi}^{\omega}\left(10^{-6}\right)$ Ref. [1] \\
\hline CMD-2 2003 & $0.95 \pm 0.18$ & - \\
CMD-2 2006 & $1.02 \pm 0.09$ & - \\
SND & $1.22 \pm 0.07$ & - \\
CMD-2 + SND & $1.13 \pm 0.05$ & $1.22 \pm 0.04$ \\
BABAR & $1.05 \pm 0.08$ & $1.78 \pm 0.01$ \\
\hline
\end{tabular}

discrepancy for BABAR. Whereas the value from the published BABAR fit to the pion form factor agrees well with Novosibirsk, the value from Ref. [1] is problematic: it devi- ates considerably from the direct value and the quoted uncertainty is one order of magnitude too small, given the statistical accuracy of the BABAR data. Thus the conclusion 


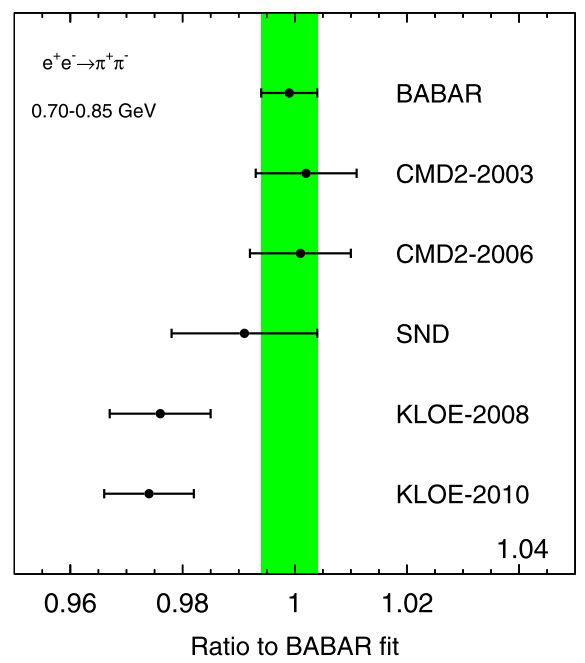

Fig. 2 Ratios of the measured $e^{+} e^{-} \rightarrow \pi^{+} \pi^{-}$cross sections from the quoted experiments relative to the BABAR cross section fit in the mass range $0.7-0.85 \mathrm{GeV}$, excluding the $\rho-\omega$ interference region. The quoted error bars include both statistical and systematic uncertainties

reached in Ref. [1] that the BABAR data strongly disagree with the Novosibirsk experiments is ill-founded.

\section{The muon magnetic anomaly}

The authors of Ref. [1] present a range of values indicating a discrepancy with the direct measurement [10], with their 'best' estimate given at $4.9 \sigma$. This result follows from two facts: the prediction has a lower central value, as the result of discarding the BABAR data, and its uncertainty is reduced because their model brings additional constraints.

In comparison the most recent analyses [20, 21] are model-independent and use all the available $e^{+} e^{-}$data, taking into account the existing discrepancies between experiments in order to set realistic uncertainties. In that case the discrepancy in the muon magnetic anomaly is only at $3.3-3.7 \sigma$.

\section{Conclusions}

We have pointed out serious discrepancies in the modeldependent analysis of Ref. [1] when compared to the original $e^{+} e^{-} \rightarrow \pi^{+} \pi^{-}$cross section data. Let us summarize our points:
- The approach relies crucially on model-dependent isospin-breaking corrections involved in relating the $\tau$ spectral function to the $e^{+} e^{-}$cross section. A detailed study of the corresponding systematic uncertainties, mandatory before using the model to discriminate between various data sets, is lacking.

- The direct and quantitative comparison of the different $e^{+} e^{-}$data sets contradicts the claim made that BABAR data disagree with the other experiments.

- The direct BABAR analysis of $\rho-\omega$ interference gives a result in agreement with Novosibirsk data, in contradiction with the value given in Ref. [1] which makes no sense and is again used to discriminate against the BABAR data.

- As a consequence we do not believe that the lower value and the smaller uncertainty obtained for the prediction of the muon magnetic anomaly are reliable results.

Open Access This article is distributed under the terms of the Creative Commons Attribution License which permits any use, distribution, and reproduction in any medium, provided the original author(s) and the source are credited.

\section{References}

1. M. Benayoun et al., Eur. Phys. J. C 73, 2453 (2013). arXiv:1210. $7184 \mathrm{v} 3$

2. M. Fujikawa et al., Phys. Rev. D 78, 072006 (2008)

3. F. Ambrosino et al., Phys. Lett. B 670, 285 (2009)

4. F. Ambrosino et al., Phys. Lett. B 700, 102-110 (2011)

5. R.R. Akhmetshin et al., Phys. Lett. B 578, 285 (2004)

6. R.R. Akhmetshin et al., Phys. Lett. B 648, 28 (2007)

7. M.N. Achasov et al., J. Exp. Theor. Phys. 103, 380 (2006)

8. B. Aubert et al., Phys. Rev. Lett. 103, 231801 (2009)

9. J.P. Lees et al., Phys. Rev. D 86, 032013 (2012)

10. G.W. Bennett et al., Phys. Rev. D 73, 072003 (2006)

11. R. Alemany, M. Davier, A. Hocker, Eur. Phys. J. C 2, 123 (1998)

12. V. Cirigliano, G. Ecker, H. Neufeld, Phys. Lett. B 513, 361 (2001)

13. V. Cirigliano, G. Ecker, H. Neufeld, J. High Energy Phys. 08, 002 (2002)

14. M. Davier, S. Eidelman, A. Höcker, Z. Zhang, Eur. Phys. J. C 27, 497 (2003)

15. M. Davier, S. Eidelman, A. Höcker, Z. Zhang, Eur. Phys. J. C 31, $503(2003)$

16. A. Flores-Talpa et al., Phys. Rev. D 74, 071301 (2006)

17. A. Flores-Talpa et al., Nucl. Phys. B, Proc. Suppl. 169, 250 (2007)

18. M. Davier et al., Eur. Phys. J. C 66, 127 (2010)

19. F. Jegerlehner, R. Szafron, Eur. Phys. J. C 71, 1632 (2011)

20. M. Davier, A. Hoecker, B. Malaescu, Z. Zhang, Eur. Phys. J. C 71, 1515 (2011)

21. K. Hagiwara et al., J. Phys. G 38, 085003 (2011) 